\title{
Comparison of Downlink Transmit Diversity Schemes for RAKE and SINR Maximizing Receivers
}

\author{
Massimiliano Lenardi, Abdelkader Medles and Dirk T.M. Slock \\ Mobile Communications Department - Institut Eurécom \\ 2229 Route des Crêtes, B.P. 193, F-06904 Sophia Antipolis Cedex, FRANCE \\ Tel: +33 4930026 26, Fax: +33493002627 \{lenardi,medles,slock\} @eurecom.fr
}

\begin{abstract}
In DS-CDMA communications, the conventional receiver is the RAKE receiver. In the downlink (base station to mobile) signalling with celldependent scrambling, orthogonal codes and a common channel for all the users, this receiver does not maximize the Signal-to-Interference-plus-Noise Ratio (SINR) at its output. Another receiver, with the same structure as the RAKE receiver, is suitable for downlink DS-CDMA communications; the one in which the channel matched filter gets replaced by a filter that is designed to maximize the SINR at the receiver output. In this paper, we analyze the use of three different Transmission Diversity (TD) techniques, namely Space-Time TD (STTD), Orthogonal TD (OTD) and Delay TD (DTD). All of them are compared for the two receiver structures: RAKE and max-SINR receivers. The max-SINR receiver structures proposed here for the three TD modes are new and are shown to usually significantly outperform the RAKE schemes. We also discuss the relative performance merits of the three TD schemes for one or the other receiver structure.
\end{abstract}

\section{INTRODUCTION}

3rd generation systems for wireless communications will require higher data rates and better BER. The multiple access interference being the major impairment for these systems, its cancellation is a must for the receivers to reach such performances. In Direct Sequence Code Division Multiple Access (DS-CDMA) schemes, the uplink and the downlink interference is asymmetric due to the fact that the uplink signalling is asynchronous, while the downlink (intracell) signalling is synchronous. This situation in the downlink makes worthwile the use of orthogonal codes which, in the absence of multipath propagation, allow the cancellation of the multiple access interference by a simple correlator, maximizing the output SNR if the noise is white. When delay spread and multipath propagation are present, a RAKE receiver will treat the different contributions in a maximum ratio combining fashion, maximizing the SNR but destroying the orthogonality between intracell user codes, leading to intracell interference at its output. In [1], a channel equalization approach has been studied, focusing on the intracell interference rather then on noise and intercell interference cancellation. Due to oversampling w.r.t. to chip rate (or multiple antennas), some of the intercell interference can be also cancelled by using the excess bandwidth. Since the orthogonality is restored by an equalizer, a simple correlator gets rid of the intracell interference. This approach has the disadvantage to enhance the noise much more then the RAKE approach, so that, between the two structures, one is better than the other depending on whether the intracell interference is higher or lower than the intercell interference plus noise. In [2], a solution to improve the RAKE and the equalizer approaches is proposed and a new class of linear receivers is introduced. These receivers have the same structure as a RAKE receiver, where the channel macthed filter gets replaced by an equalizer filter designed to maximize the SINR at the receiver output. The idea behind this ap-

Eurécom's research is partially supported by its industrial partners: Ascom, Swisscom, ThomsonCSF, IBM France, CEGETEL, Motorola, France Télécom, Hitachi Europe and Texas Instruments. The work leading to this paper was also partially supported by the French RNRT (National Network for Telecommunications Research) project AUBE. The research of A. Medles was also sponsored by the research contract Multisensor Antennas of the "Credits Incitatifs" programme of the Groupe des Ecoles de Telecommunications (GET), France. proach is that when a cell-dependent scrambler is superposed to the periodic spreading codes, the received signal is stationary if sampled at chip rate; therefore, a time-invariant MMSE design is feasible for the equalizer and it turns out to lead to the max-SINR receiver. The filter adaptation can be done at symbol or chip rate.

Multiple transmitting antennas at the base station can improve performances due to increase in diversity and some schemes have been proposed for open loop systems (no knowledge of the downlink channel at the transmitter). Basically, 4 kinds of Transmission Diversity (TD) schemes have been proposed for a base station: Orthogonal TD (OTD, see [3]), Space-Time TD (STTD, see [4]), Time-Switched TD (TSTD, see [3]) and Delay TD (DTD, see [5]). The UMTS norm for 3rd generation wireless systems specifies, for the FDD downlink, that the use of Trasmission Diversity techniques is optional at the base station, while it is mandatory for the mobile station. In this paper, we analyze the use of three TD techniques, namely STTD, OTD and DTD. All of them are compared for the two receiver structures, RAKE and max-SINR receivers.

\section{BS TRANSMISSION DIVERSITY SCHEMES}

Fig. 1 shows the downlink signal model in baseband. The $K$ users are assumed to transmit linearly modulated signals over the same linear multipath channels with additive noise and intercell interference, by using two antennas $j=1,2$ at the base station. The two signals are generated following different rules given by the Transmission Diversity Schemes described later in II-A to II-C. The symbol and chip periods $T$ and $T_{c}$ are related through the spreading factor $L: T=L T_{c}$, which is assumed here to be common for all the users. The total chip sequences $b_{l}^{1}$ and $b_{l}^{2}$ are the sum of the chip sequences of all the users over the respective antenna 1 and 2. Every user chip sequence is given by the product between the $n$th symbol of the $k$ th user and an aperiodic spreading sequence $w_{k, l}$ which is itself the product of a periodic Walsh-Hadamard (with unit energy)

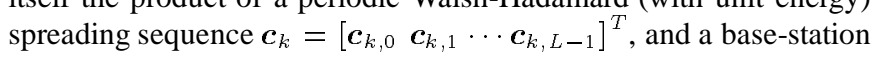
specific unit magnitude complex scrambling sequence $s_{l}$ with variance $1, w_{k, l}=c_{k, l} \bmod L s_{l}$ :

$$
b_{l}^{j}=\sum_{k=1}^{K} b_{k, l}^{j}=\sum_{k=1}^{K} a_{k,\left\lfloor\frac{l}{L}\right\rfloor}^{j} w_{k, l} \quad j=1,2 .
$$

The scrambling operation is a multiplication of chip rate sequences. The spreading operation is represented by a filtering of an upsampled symbol sequence with the spreading sequence as impulse response. The chip sequence $b_{l}^{1,2}$ get transformed into a continuous-time signals by filtering them with the pulse shape $p(t)$ and then pass through the multipath propagation channels $h^{1}(t)$ and $h^{2}(t)$ (from antenna 1 and from antenna 2 to the mobile station respectively) to yield the total received signal $y(t)$. The receiver samples $M$ times per chip the lowpass filtered received signal. Stacking the $M$ samples per chip 


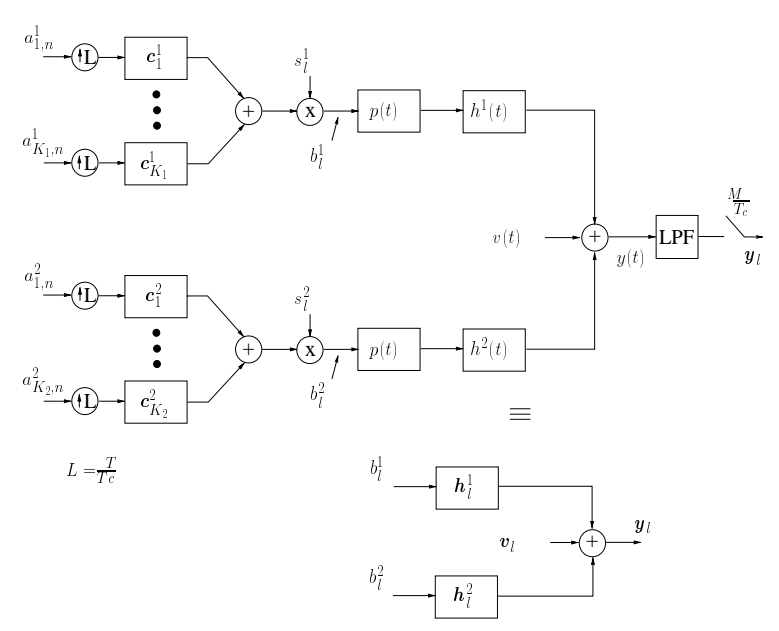

Fig. 1. Downlink signal model

period in vectors, we get for the sampled received signal

$$
\boldsymbol{y}_{l}=\boldsymbol{y}_{l}^{1}+\boldsymbol{y}_{l}^{2}+\boldsymbol{v}_{l}, \quad \boldsymbol{y}_{l}^{j}=\sum_{k=1}^{K} \sum_{i=0}^{N-1} h_{i}^{j} b_{k, l-i}^{j} \quad j=1,2
$$

$$
\text { where } \boldsymbol{y}_{l}^{j}=\left[\begin{array}{c}
y_{1, l}^{j} \\
\vdots \\
y_{M, l}^{j}
\end{array}\right], \boldsymbol{h}_{l}^{j}=\left[\begin{array}{c}
h_{1, l}^{j} \\
\vdots \\
h_{M, l}^{j}
\end{array}\right], \boldsymbol{v}_{l}=\left[\begin{array}{c}
v_{1, l} \\
\vdots \\
v_{M, l}
\end{array}\right] .
$$

Here $h_{l}^{j}$ represents the vectorized samples of the overall channel $h^{j}(t)$, including pulse shape, propagation channel and receiver filter. The overall channels $h^{j}(t)$ are assumed to have the same delay spread of $N$ chips. If we model the scrambling sequence and the symbol sequences as independent i.i.d. sequences, then the chip sequences $b_{l}^{1,2}$ are sums of $K$ independent white noises (chip rate i.i.d. sequences, hence stationary). The intracell contribution to $\boldsymbol{y}_{l}$ then is a stationary (vector) process (the continuous-time counterpart is cyclostationary with chip period). The intercell interference is a sum of contributions that are of the same form as the intracell contribution. The remaining noise is assumed to be white stationary noise. Hence the sum of intercell interference and noise, $\boldsymbol{v}_{l}$, is stationary.

\section{A. OTD SCHEME}

For each user $k$, this TD scheme generates, from a couple of symbols to transmit $a_{k, 2 P}$ and $a_{k, 2 P+1}$, where $2 P$ stays for even symbol periods, the two pairs of symbols to be sent through the two antennas as following:

$$
\begin{array}{ll}
a_{k, 2 P}^{1}=a_{k, 2 P} & a_{k, 2 P+1}^{1}=a_{k, 2 P} \\
a_{k, 2 P}^{2}=a_{k, 2 P+1} & a_{k, 2 P+1}^{2}=-a_{k, 2 P+1}
\end{array}
$$

\section{B. STTD SCHEME}

Similarly to OTD, this technique produces two pairs of symbols, but now

$$
\begin{array}{ll}
a_{k, 2 P}^{1}=a_{k, 2 P} & a_{k, 2 P+1}^{1}=a_{k, 2 P+1} \\
a_{k, 2 P}^{2}=-a_{k, 2 P+1}^{*} & a_{k, 2 P+1}^{2}=a_{k, 2 P}^{*}
\end{array}
$$

where $*$ denotes the complex conjugate operation.

\section{DTD SCHEME}

This scheme operates differently from the previous two, because it sends the same symbol sequence on the two antennas, but it introduces a delay of $D$ chip periods on the transmission on the second antenna. So it works as if there is just one antenna, but the channel is the sum of $h^{1}(t)$ and $h^{2}(t-D \cdot T c)$ :

$$
\begin{aligned}
& a_{k, n}^{1}=a_{k, n}^{2}=a_{k, n}, b_{l}^{1}=b_{l}^{2}=b_{l} \\
& h(z)=h^{1}(z)+z^{-D} \cdot h^{2}(z)
\end{aligned}
$$

where $h(z)$ represent the channel in the $z$-domain.

\section{RECEIVER STRUCTURES FOR BS TRANSMISSION DIVERSITY}

Fig. 2 shows the receiver structure in case of no Transmission Diversity; it is similar to a RAKE receiver in which the channel matched filter is replaced by a general causal chip rate filter $f_{l}$ of the same length (in chip periods) of the channel $N$, so that the output estimate is delayed by a certain number of symbols $l_{1}$. As in the RAKE, the filter $f_{l}$ is followed by a descrambler (delayed by some chips $d=l_{1} L+l_{2}=N-1, l_{1}=\left\lfloor\frac{d}{L}\right\rfloor, l_{2}=d \bmod L$, and by a decorrelator for the user of interest (assumed here the user 1). Descrambling and despreading can be considered as a unique filtering with input at chip rate $\left(\hat{b}_{l-d}\right)$ and output at symbol rate (the output estimate).

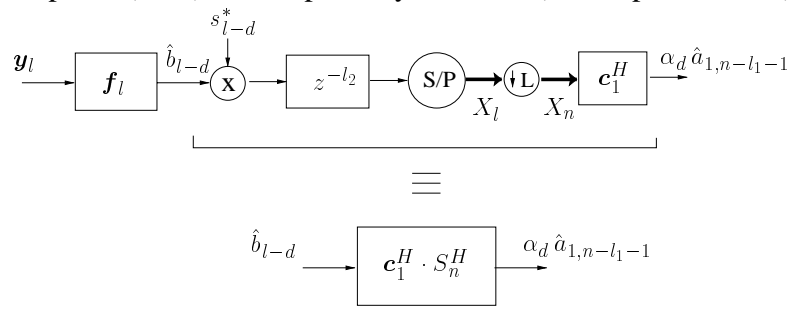

Fig. 2. The downlink receiver structure

The receiver outputs

$$
\alpha_{d} \widehat{a}_{1, n-l_{1}-1}=\boldsymbol{c}_{1}^{H} X_{n}
$$

where $X_{n}$ is a vector of descrambled filter outputs,

$$
X_{n}=S_{n-l_{1}-1}^{H} Z_{n}, \quad Z_{n}=\mathcal{T}(f) \boldsymbol{Y}_{n},
$$

$Z_{n}$ is a vector of filter outputs, $S_{n}=\operatorname{diag}\left\{s_{n, L-1}, \ldots, s_{n, 1}, s_{n, 0}\right\}$ is a diagonal matrix of scrambling code coefficients $s_{n, l}=$ $s_{n L+l}, \mathcal{T}(f)$ is the block Toeplitz filtering matrix with $f=$ $\left[f_{0} \cdots f_{N-1}\right]$ (padded with zeros) as first block row, and $\boldsymbol{Y}_{n}=\left[\underline{Y}_{n, l_{2}}^{T} Y_{n-1}^{T} \cdots Y_{n-l_{3}}^{T} \bar{Y}_{n-l_{3}-1, l_{4}}^{T}\right]^{T}$ where $N+L-1-l_{2}=$ $l_{3} L+l_{4}, Y_{n}=\left[\boldsymbol{y}_{n, L-1}^{T} \cdots \boldsymbol{y}_{n, 0}^{T}\right]^{T}, \underline{Y}_{n, l}=\left[\boldsymbol{y}_{n, l-1}^{T} \cdots \boldsymbol{y}_{n, 0}^{T}\right]^{T}$, $\bar{Y}_{n, l}=\left[\boldsymbol{y}_{n, L-1}^{T} \cdots \boldsymbol{y}_{n, L-l}^{T}\right]^{T}$, and $\boldsymbol{y}_{n, l}=\boldsymbol{y}_{n L+l}$. The structure of the vector $\boldsymbol{Y}_{n}$ of received data that contribute to the estimate $\widehat{a}_{1, n-l_{1}-1}$ is

$$
\boldsymbol{Y}_{n}=\mathcal{T}\left(h^{\prime}\right) \boldsymbol{S}_{n} \sum_{k=1}^{K} \boldsymbol{C}_{k} \boldsymbol{A}_{k, n}+\boldsymbol{V}_{n}
$$

where $\mathcal{T}\left(h^{\prime}\right)$ is again a block Toeplitz filtering matrix with the zero padded $h^{\prime}=\left[h_{0} \cdots h_{N-1}\right]$ as first block row, $\boldsymbol{S}_{n}=$ blockdiag $\left\{\underline{S}_{n, l_{2}}, S_{n-1}, \ldots, S_{n-l_{5}}, \bar{S}_{n-l_{5}-1, l_{6}}\right\}$ is the scrambling

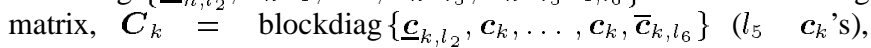
$\boldsymbol{A}_{k, n}=\left[a_{k, n} \cdots a_{k, n-l_{5}-1}\right]^{T}, \boldsymbol{V}_{n}$ is defined like $\boldsymbol{Y}_{n}$, and $\underline{S}_{n, l}$, $\bar{S}_{n, l}, \underline{\boldsymbol{c}}_{k, l}$ and $\overline{\boldsymbol{c}}_{k, l}$ are defined similarly to $\underline{Y}_{n, l}$ and $\bar{Y}_{n, l}$ except that $\underline{S}_{n, l}$ and $\bar{S}_{n, l}$ are diagonal matrices, and $2 N+L-2-l_{2}=l_{5} L+l_{6}$. We have for the filter-channel cascade

$$
\mathcal{T}(f) \mathcal{T}(h)=\mathcal{T}(\boldsymbol{\alpha})=\mathcal{T}\left(\boldsymbol{\alpha}_{d}\right)+\mathcal{T}\left(\overline{\boldsymbol{\alpha}}_{d}\right)
$$


where

$$
\begin{aligned}
& \boldsymbol{\alpha}=\left[\alpha_{0} \cdots \alpha_{2 N-2}\right], \boldsymbol{\alpha}_{d}=\left[0 \cdots 0 \alpha_{d} 0 \cdots 0\right] \\
& \overline{\boldsymbol{\alpha}}_{d}=\left[\begin{array}{lll}
\alpha_{0} \cdots \alpha_{d-1} & 0 & \alpha_{d+1} \cdots \alpha_{2 N-2}
\end{array}\right] \text {. }
\end{aligned}
$$

In the noiseless case (and no intercell interference), the use of a ZF equalizer leads to $\overline{\boldsymbol{\alpha}}_{d}=[0 \cdots 0]$ and $\widehat{a}_{1, n-l_{1}-1}=a_{1, n-l_{1}-1}\left(\alpha_{d}=\right.$ 1). A RAKE receiver corresponds to $f=h^{H}, \alpha_{d}=\|\boldsymbol{h}\|^{2}$, where $h=\left[h_{N-1}^{T} \cdots h_{0}^{T}\right]^{T}$.

In [2] is presented the general expression for the SINR at the output of a receiver when no BS Transmission Diversity is used, namely $\Gamma=$ $\frac{\sigma_{1}^{2}\left|\alpha_{d}\right|^{2}}{M S E}$ or

$$
\Gamma=\frac{\sigma_{1}^{2}\left|\alpha_{d}\right|^{2}}{f A f^{H}-\sigma_{t o t}^{2}\left|\alpha_{d}\right|^{2}}
$$

where $A=R_{V V}+\sigma_{\text {tot }}^{2} \mathcal{T}\left(h^{\prime}\right) \mathcal{T}^{H}\left(h^{\prime}\right), R_{V V}=\mathrm{E} V_{n} V_{n}^{H}$ and $\sigma_{\text {tot }}^{2}=\frac{1}{L} \sum_{k=1}^{K} \sigma_{k}^{2}$. The filter $f$ that maximizes the receiver output SINR is unique up to a scale factor and comes from the following problem

$$
f_{M A X}=\arg \max _{f: f h=1} \Gamma=\arg \min _{f: f h=1} f A f^{H} .
$$

The solution is $\left(\alpha_{d}=1\right)$

$$
f_{M A X}=\left(h^{H} A^{-1} h\right)^{-1} h^{H} A^{-1}
$$

and the maximum SINR becomes $\left(\alpha_{d}^{M A X}=1\right)$

$$
\Gamma_{M A X}=\frac{\sigma_{1}^{2}}{\left(h^{H} A^{-1} h\right)^{-1}-\sigma_{t o t}^{2}}
$$

A turns out to be the covariance matrix of the received signal, while $f_{\text {MAX }}$ to be the unbiased MMSE receiver. The max-SINR receiver is therefore a cascade of an (unbiased if $\alpha_{d}=1$ ) MMSE receiver for the desired user's chip sequence, followed by a descrambler and a correlator. In the noiseless case, the MMSE receiver $f_{M A X}$ becomes a ZF equalizer.

\section{A. DTD RECEIVER}

The structure in Fig. 2 is valid also in the case of Delay Transmission Diversity. Eq. (7) to Eq. (15) are also applicable for this TD scheme, when the channel $h$ is as in the Eq. (6). The same results and conclusions are then valid, in particular, in the noiseless case, the max-SINR receiver becomes the ZF equalizer.

\section{B. OTD RECEIVER}

When other schemes of Transmission Diversity are used, other structures are needed. The received signal in this case can be expressed as

$$
\boldsymbol{Y}_{n}=\boldsymbol{Y}_{n}^{1}+\boldsymbol{Y}_{n}^{2}+\boldsymbol{V}_{n}
$$

where

$$
\begin{aligned}
& \boldsymbol{Y}_{n}^{1}=\mathcal{T}\left(h^{1^{\prime}}\right) \boldsymbol{S}_{n} \sum_{k=1}^{K} \boldsymbol{C}_{k} \boldsymbol{A}_{k, n}^{1} \\
& \boldsymbol{Y}_{n}^{2}=\mathcal{T}\left(h^{2^{\prime}}\right) \boldsymbol{S}_{n} \sum_{k=1}^{K} \boldsymbol{C}_{k} \boldsymbol{A}_{k, n}^{2}
\end{aligned}
$$

where notations are the same as above but with superscripts 1,2 .

Fig. 3 shows the linear processing needed in an OTD receiver, which distinguishes even $(2 P)$ and odd $(2 P+1)$ symbol periods within the received signal $Y_{n}$. The receiver processes the two signals separetely with two chip rate filters $f_{1}$ and $f_{2}$, whose outputs are then despreaded, by applying a (total) despreader similarly to what is shown in Fig.2. When this scheme is implemented, the two softoutputs (at half of the symbol rate) are the estimators for even symbol period $\left(z^{1}\right)$ and odd symbol period $\left(z^{2}\right)$ where $\alpha_{d}^{j \bar{j}}$ is defined as in

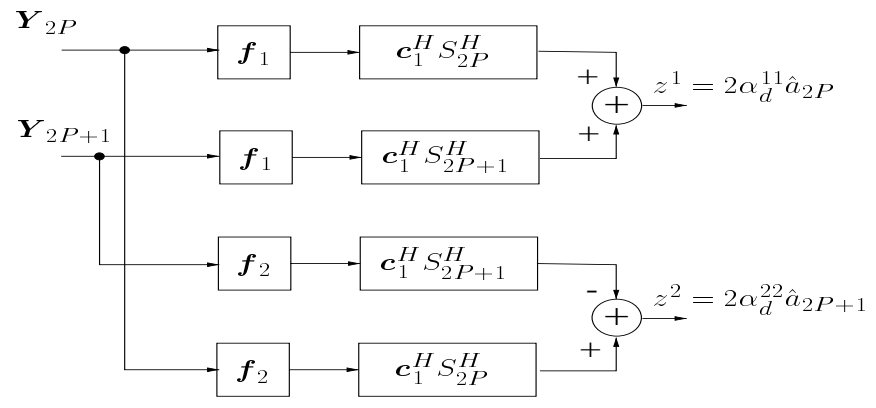

Fig. 3. The downlink receiver OTD structure

Eq. (10) and Eq. (11) for filter $f_{j}$ and channel $h^{\bar{j}}$ :

$$
\alpha_{d}^{j \bar{j}}=f_{j} h^{\bar{j}} \text {. }
$$

We can write the two softoutputs $z^{j}$ as

$$
\begin{aligned}
z^{1} & =\boldsymbol{c}_{1}^{H} S_{2 P}^{H} \mathcal{T}\left(\boldsymbol{f}_{1}\right) \boldsymbol{Y}_{2 P}+\boldsymbol{c}_{1}^{H} S_{2 P+1}^{H} \mathcal{T}\left(\boldsymbol{f}_{1}\right) \boldsymbol{Y}_{2 P+1} \\
z^{2} & =\boldsymbol{c}_{1}^{H} S_{2 P}^{H} \mathcal{T}\left(\boldsymbol{f}_{2}\right) \boldsymbol{Y}_{2 P}-\boldsymbol{c}_{1}^{H} S_{2 P+1}^{H} \mathcal{T}\left(\boldsymbol{f}_{2}\right) \boldsymbol{Y}_{2 P+1}
\end{aligned}
$$

When a RAKE implementation is wanted, then $f_{1}=h^{1^{H}}$ and $\boldsymbol{f}_{2}=$ $h^{2^{H}}, \alpha_{d}^{11}=\left\|h^{1}\right\|^{2}$ and $\alpha_{d}^{22}=\left\|h^{2}\right\|^{2}$. The SINR for the softoutput $z_{j}$ is

where

$$
\Gamma_{j}=\frac{\sigma_{1}^{2}\left|\alpha_{d}^{j j}\right|^{2}}{2 \cdot f_{j} A_{1} f_{j}^{H}}
$$

$$
\begin{aligned}
A_{1}= & R_{V V}+\sigma_{\text {tot }}^{2}\left[\mathcal{T}\left(h^{1^{\prime}}\right) \mathcal{T}^{H}\left(h^{1^{\prime}}\right)+\mathcal{T}\left(h^{2^{\prime}}\right) \mathcal{T}^{H}\left(h^{2^{\prime}}\right)-\right. \\
& \left.-\left(h^{1} h^{1^{H}}+h^{2} h^{2^{H}}\right)\right]
\end{aligned}
$$

From the OTD receiver structure is clear that the maximization of $\Gamma_{1}$ and $\Gamma_{2}$ is independent ( $z_{1}$ depends only on $f_{1}$ and $z_{2}$ only on $f_{2}$ ). The expression for the filters turns out to be the solution of

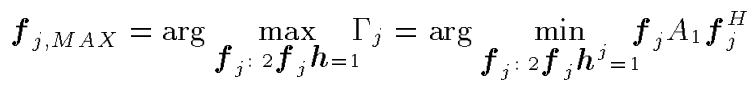

that is

$$
f_{j, M A X}=\left(h^{j^{H}} A_{1}^{-1} h^{j}\right)^{-1} h^{j^{H}} A_{1}^{-1}
$$

In this case the maximum SINR for softoutput $z_{j}$ becomes

$$
\Gamma_{j, M A X}=2 \cdot \sigma_{1}^{2} \cdot h^{j^{H}} A_{1}^{-1} h^{j} .
$$

$\Gamma_{1, M A X}$ and $\Gamma_{2, M A X}$ are not equal so the transmission quality of the two equivalent channels is different. The total SINR for OTD is then defined as $\Gamma_{M A X, O T D}=2\left(\frac{1}{\Gamma_{1, M A X}}+\frac{1}{\Gamma_{2, M A X}}\right)^{-1}$ which corresponds to take the MSE as the average of the MSEs for the two softouputs.

\section{STTD RECEIVER}

Fig. 4 shows the STTD receiver structure; now each of the two softoutput depends on both $\boldsymbol{f}_{j}$ and the two input signals $Y_{2 P}$ and $Y_{2 P+1}$ are complex conjugated before being filtered by $f_{2}$. We can write the two STTD softoutputs $z^{j}$ as

$$
\begin{aligned}
& z^{1}=\boldsymbol{c}_{1}^{H} S_{2 P}^{H} \mathcal{T}\left(\boldsymbol{f}_{1}\right) \boldsymbol{Y}_{2 P}+\boldsymbol{c}_{1}^{T} S_{2 P+1}^{T} \mathcal{T}\left(\boldsymbol{f}_{2}\right) \boldsymbol{Y}_{2 P+1}^{*} \\
& z^{2}=\boldsymbol{c}_{1}^{H} S_{2 P+1}^{H} \mathcal{T}\left(\boldsymbol{f}_{1}\right) \boldsymbol{Y}_{2 P+1}-\boldsymbol{c}_{1}^{T} S_{2 P}^{T} \mathcal{T}\left(\boldsymbol{f}_{2}\right) \boldsymbol{Y}_{2 P}^{*}
\end{aligned}
$$




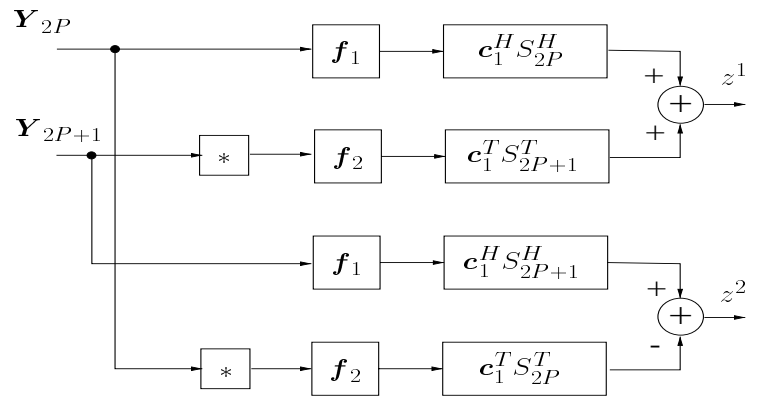

Fig. 4. The downlink receiver STTD structure

By defining $\boldsymbol{F}=\left[\boldsymbol{f}_{1}, \boldsymbol{f}_{2}\right], \boldsymbol{H}_{1}=\left[\boldsymbol{h}^{1^{T}}, \boldsymbol{h}^{2^{H}}\right]^{T}$ and $\boldsymbol{H}_{2}=$ $\left[-h^{2^{T}}, h^{1^{H}}\right]^{T}$ and by taking the expectation over the despreading/descrambling, we can restate the two softoutputs estimates $z^{1}$ and $z^{1}$ as

$$
\begin{aligned}
& z^{1}=\boldsymbol{F} \boldsymbol{H}_{1} \hat{a}_{2 P}+\boldsymbol{F} \boldsymbol{H}_{2} \hat{a}_{2 P+1}^{*} \\
& z^{2}=\boldsymbol{F} \boldsymbol{H}_{1} \hat{a}_{2 P+1}-\boldsymbol{F} \boldsymbol{H}_{2} \hat{a}_{2 P}^{*}
\end{aligned}
$$

and the SINR for the $z^{j}$ sofoutput becomes

$$
\Gamma_{j}=\frac{\sigma_{1}^{2}\left(\left|\boldsymbol{F} \boldsymbol{H}_{1}\right|^{2}+\left|\boldsymbol{F} \boldsymbol{H}_{2}\right|^{2}\right)}{\boldsymbol{F} A \boldsymbol{F}^{H}+R_{j}}
$$

where $A=\left(\begin{array}{cc}A_{1} & 0 \\ 0 & A_{1}^{*}\end{array}\right)$ and $A_{1}$ is defined in Eq. (21). The term $R_{j}$ in the denumerator depends mainly on the used spreading codes (so the mobile receiver should know them), but we will see in section IV that it is negligible with respect to the other term, so that, from Eq. (27), $\Gamma_{1}=\Gamma_{2}$ and

$$
\Gamma_{S T T D}=\frac{\sigma_{1}^{2}\left(\left|\boldsymbol{F} \boldsymbol{H}_{1}\right|^{2}+\left|\boldsymbol{F} \boldsymbol{H}_{2}\right|^{2}\right)}{\boldsymbol{F} A \boldsymbol{F}^{H}}
$$

Since we want $z^{1}$ to be the estimator for $a_{2 P}$ and $z^{2}$ the one for $a_{2 P+1}$, the filter $\boldsymbol{F}_{M A X}$ that maximize $\Gamma_{S T T D}$ comes from the problem

$$
\begin{aligned}
& \arg \max \Gamma_{S T T D}=\arg \min \boldsymbol{F} A \boldsymbol{F}^{H}
\end{aligned}
$$

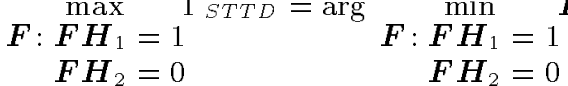

that is

$$
\boldsymbol{F}_{M A X}=\left(\boldsymbol{H}_{1}^{H} A^{-1} \boldsymbol{H}_{1}\right)^{-1} \boldsymbol{H}_{1}^{H} A^{-1} .
$$

In this case the maximum SINR becomes

$$
\Gamma_{M A X, S T T D}=\sigma_{1}^{2}\left(h^{1^{H}} A_{1}^{-1} h^{1}+h^{2^{H}} A_{1}^{-1} h^{2}\right) .
$$

When a RAKE receiver is implemented, then $\boldsymbol{F}=\left[\boldsymbol{h}^{1^{H}}, \boldsymbol{h}^{2^{T}}\right]$, $\boldsymbol{F} \boldsymbol{H}_{1}=\left(\left\|\boldsymbol{h}^{1}\right\|^{2}+\left\|\boldsymbol{h}^{2}\right\|^{2}\right)$ and $\boldsymbol{F} \boldsymbol{H}_{2}=0$.

\section{NUMERICAL EXAMPLES}

Fig. 5 to Fig. 9 present some of the simulations that we have performed to evaluate the various schemes and receivers. In the legends of these figures, $\mathrm{R}$ and MS refer to RAKE and max-SINR receiver respectively. The $K$ users are considered synchronous, with the same spreading factor $L=32$ and using the same downlink channels $h^{1}$ and $h^{2}$ which are FIR filters, convolution of a sparse Vehicular A UMTS channel and a pulse shape (root-raised cosine with roll-off factor of 0.22 ). The channel(s) length is $N=19$ chips, due to the UMTS chip rate of $3.84 \mathrm{Mchips} / \mathrm{sec}$. An oversampling factor of $M=2$ is assumed. Two possible user power distributions are simulated: all interferers have the same power and the user of interest has either the same power also or $15 \mathrm{~dB}$ less power (near-far situation).

The performances of the different receiver instances are shown in terms of the output SINR versus the SNR at the receiver. The length of all the filters in the simulations is equal for all the TD schemes and is either the channel length $N$ or the channel length of the DTD channel $(N+D)$.

Due to the interference between the two channels (see Eq. (21)) and to the presence of the the scrambler, it is clear that a ZF equalization for OTD and STTD can not exist; this is confirmed by the simulations in Fig. 5 to Fig. 8, where there is always SINR saturation for OTD and STTD structures, while DTD is saturating for shorter FIR filters because their length does not permit zero forcing. In this set of figures the delay $D$ for DTD is equal to the channel length $N$.

We can notice how the DTD max-SINR receiver performs much better than the other structures for both user power distributions when the filter lengths coincide with the DTD channel length $(2 N)$; see Fig. 5 and Fig. 6. The DTD RAKE implementation is also the best one in these cases.

When, instead, the filter lengths are taken equal to the channel length $N$, but maintaining $D=N$, the DTD receivers clearly suffer. In this case STTD performs better in the RAKE implementation (see Fig. 7 and Fig. 8) and besides the DTD complexity is here half of the STTD complexity. Their max-SINR performances are similar.

Fig. 9 shows the case when the delay $D$ is taken as half the channel length $N$ and the filter lengths are equal to $N+D$ (so the complexity is reduced by one quarter). The performances are very similar to those in Fig. 5.

The last figure, Fig. 10, is shown to confirm that the terms $R_{j}$ in Eq. (27) are negligible. We can see that when they are taken into account (x's and sparse dots), the performance/results are identical, on the average, to the case when they are dropped (dashed and solid lines respectively).

\section{CONCLUSIONS}

The RAKE performs best with the DTD scheme, regardless of how much delay is introduced between the two channels (hence even if only partial diversity). Nevertheless, lesser temporal overlap between the two channels in DTD leads to better performance. When a max-SINR receiver is employed, performance still gets improved significantly for DTD, compared to a RAKE receiver. The good performance of the DTD scheme can be explained by the fact that it is the only TD scheme that allows zero-forcing equalization. STTD schemes often perform significantly worse than DTD schemes, though they may occasionally outperform DTD schemes a bit. We can also conclude that the OTD scheme leads to the worst performance in all receiver cases.

\section{REFERENCES}

[1] I. Ghauri and D. T. M. Slock, "Linear receivers for the DS-CDMA downlink exploiting orthogonality of spreading sequences," in Proc. 32nd Asilomar Conf. on Signals, Systems \& Computers, (Pacific Grove, CA), November 1998.

[2] M. Lenardi and D. T. M. Slock, "A RAKE Receiver with Intracell Interference Cancellation for a DS-CDMA Synchronous Downlink with Orthogonal Codes," in Proc. VTC 2000, (Tokyo, Japan), May 2000.

[3] "Space Time Block Coded Transmit Antenna Diversity for WCDMA," tech. rep., Texas Instruments, November 1998. Contribution to 3GPP standardization.

[4] S. M. Alamouti, "A simple transmit diversity technique for wireless communications," IEEE JSAC, vol. 16, pp. 1451-1458, October 1998.

[5] A. Wittneben, "A new bandwidth efficient transmit antenna modulation diversity scheme for linear digital modulation," in Proc. IEEE International Conf. Communications (ICC'93), (Atlanta, GA), pp. 1630-1634, May 1993. 


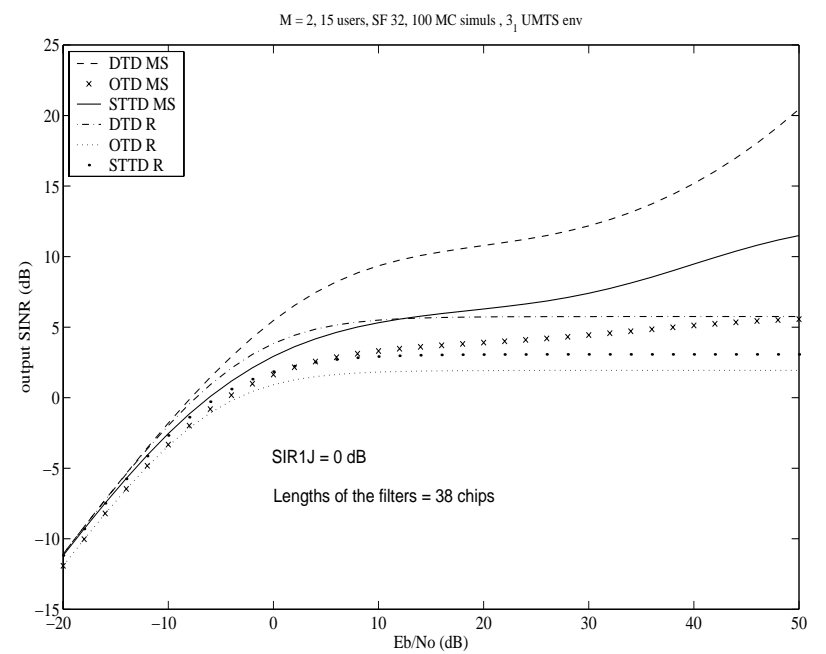

Fig. 5. Theoretical output SINR versus SNR, 50\% loaded system, spreading factor 32 and equal power distribution

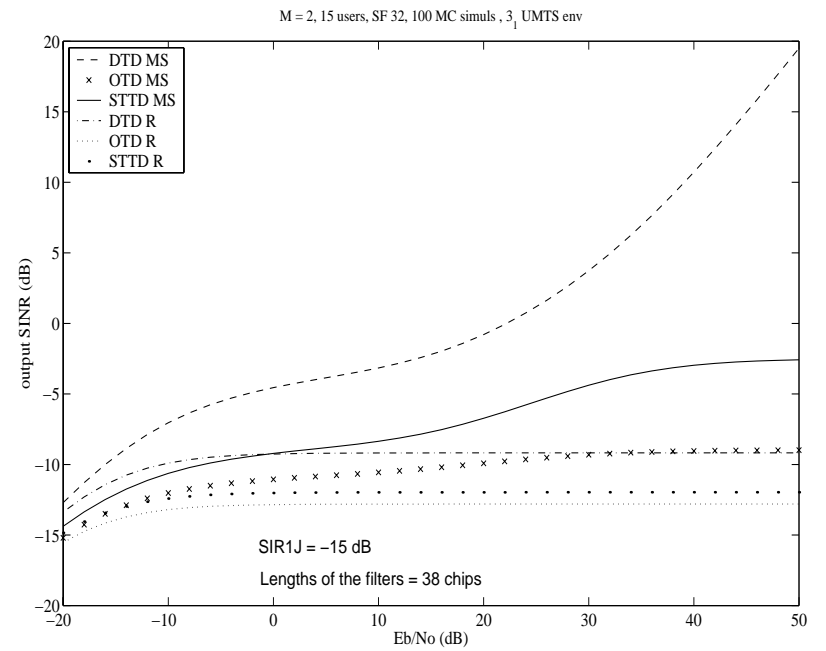

Fig. 6. Theoretical output SINR versus SNR, 50\% loaded system, spreading factor 32 and near-far situation

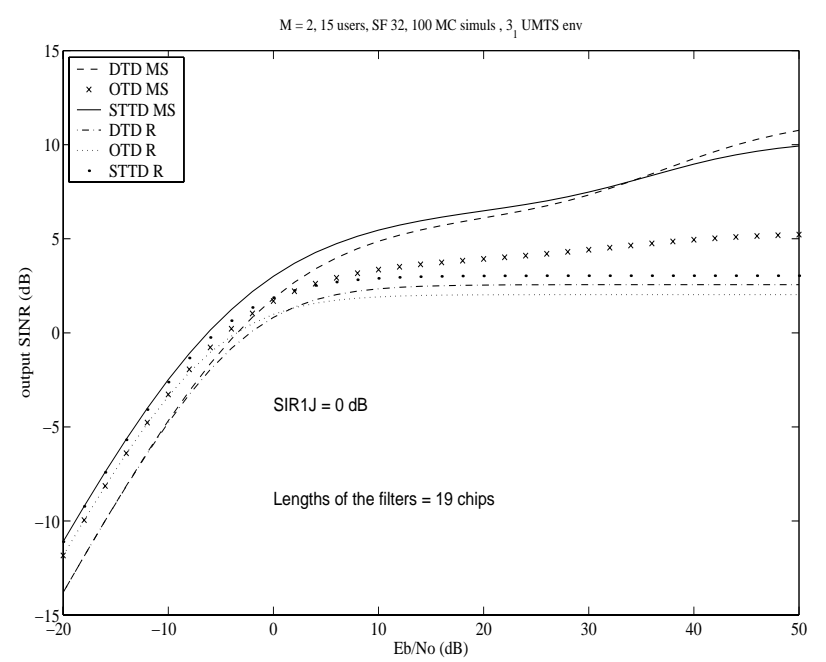

Fig. 7. Theoretical output SINR versus SNR, $50 \%$ loaded system, spreading factor 32 and equal power distribution

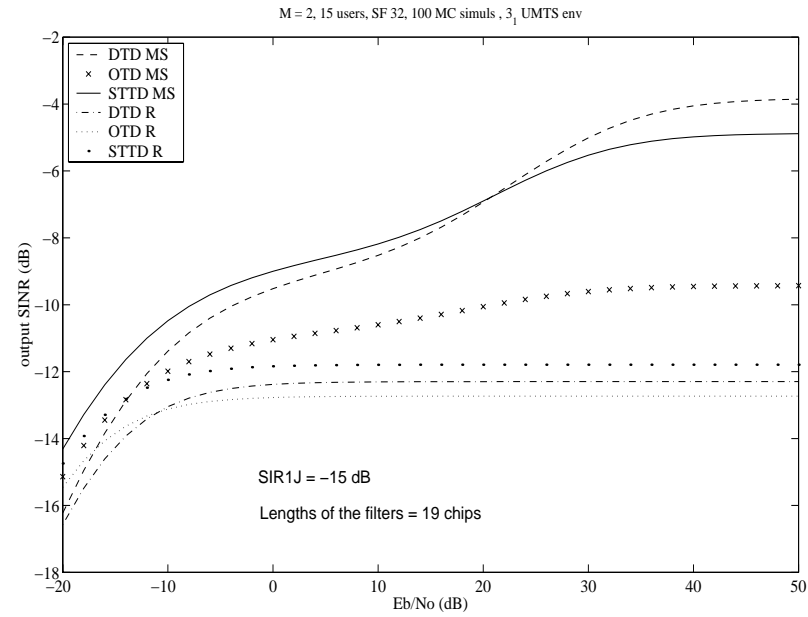

Fig. 8. Theoretical output SINR versus SNR, 50\% loaded system, spreading factor 32 and near-far situation

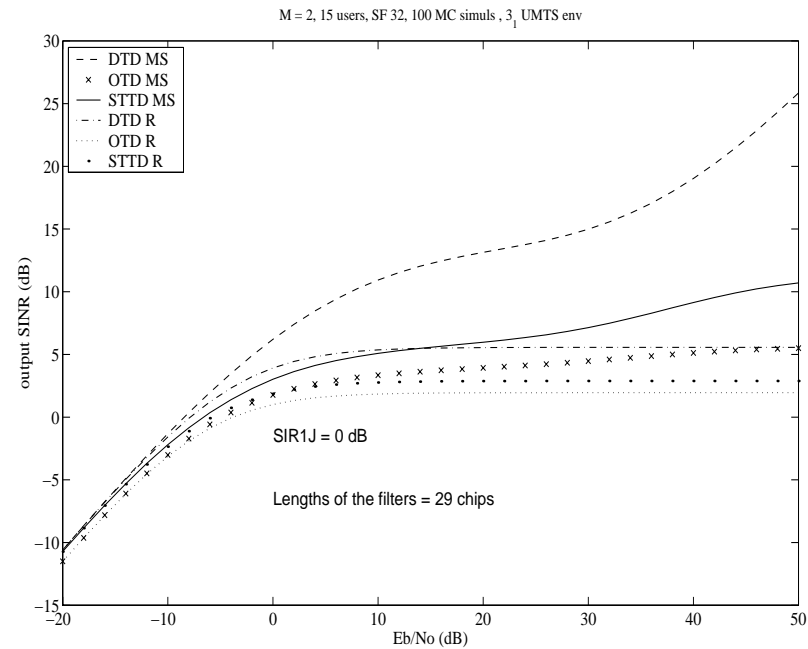

Fig. 9. Theoretical output SINR versus SNR, 50\% loaded system, spreading factor 32 and equal power distribution

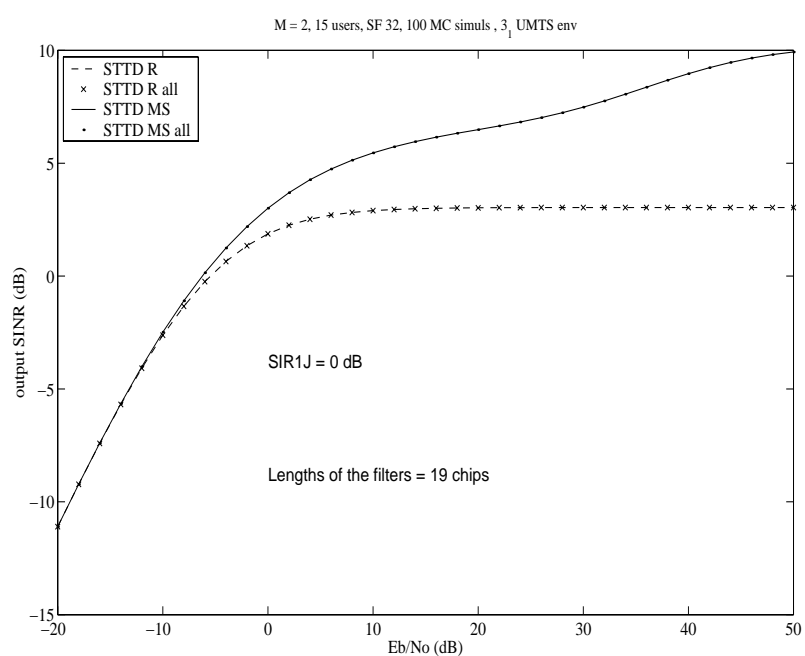

Fig. 10. Theoretical output SINR versus SNR, 50\% loaded system, spreading factor 32 and equal power distribution, STTD case only 